\title{
Super-Selective Angiography and Embolization of Skull Base Tumours
}

\author{
K.G. Ter Brugge, P. Lasjaunias and M.C. Chiu
}

\begin{abstract}
Treatment planning for patients with tumours of the skull requires a true multidisciplinary approach. Super-selective angiography, using a protocol approach, is necessary in building a proper strategy plan for treatment of these lesions. Embolization done by a well trained team has proven to be a valuable adjunct to the various treatment methods and it may eliminate the need for radiation of benign lesions of the base of the skull.
\end{abstract}

RÉSUMÉ: Angiographie super-sélective et embolisation des tumeurs de la base du crâne La planification du traitement des tumeurs du crâne demande une approche vraiment multidisciplinaire, telle que l'angiographie super-sélective. L'embolisation faite par une équipe bien entraînée s'est avérée utile comme complément aux diverses approches thérapeutiques et peut même éliminer la nécessité d’irradier les lésions bénignes de la base du crâne.

Can. J. Neurol. Sci. 1985: 12:341-344

Tumours of the base of the skull represent a diagnostic and therapeutic challenge. Because of the complex topography and often inaccessible location of such lesions, a multidisciplinary approach is required, and often the radiologist forms the necessary link between the different specialties including neurology, neurosurgery, head and neck surgery and otolaryngology.

It is, therefore, necessary that the neuroradiologist understands the relevant clinical problems in each case, and conducts the investigations with these in mind. After all the tests have been completed, there must be a clear understanding of the extent of the lesion, the blood supply of the lesion and of the adjacent normal tissues, as well as possible adverse effects of endovascular treatment on the blood supply to and from the brain and cranial nerves.

In order to properly evaluate these lesions, complete and detailed knowledge of the bony and soft tissue anatomy of the base of the skull, including the blood supply and its variations, is mandatory. Of great practical importance is an analysis of the vascularization of the dura, the base of the skull proper, and the cranial nerves, which may give crucial information with regard to therapeutic decisions.

\section{Angiography and Embolization}

Angiography is central to the investigation and treatment of tumours of the base of the skull. Clinical information and topo- graphic data obtained by plain skull films, computed tomography, and magnetic resonance imaging are necessary to build a proper angiographic protocol. ${ }^{1-6}$ Although the resolution of digital subtraction angiography (DSA) has improved in the past few years, it still is inferior to the quality of conventional manification subtraction techniques. Tumour blushes, however, may be more easily seen with DSA. The territorial blood supply of the tumour must be analyzed in order to build a three-dimensional "puzzle" of the area involved, where each piece of the puzzle is a vascular territory corresponding to a part of the tumour and a potential area of tumoral invasion. ${ }^{7,8}$

Routine super-selective catheterization of secondary and tertiary branches of the external carotid artery is now possible using the femoral approach and small size catheters (4 French, or less), and is not associated with significant risk when performed by a well trained team. The examination is usually done under general anesthesia in order to obtain the best possible circumstances for both diagnosis and treatment. A step by step protocol, as described by one of the authors, ${ }^{7}$ is routinely followed to completely demonstrate the blood supply to the lesion and the adjacent tissues. Anatomical variations are looked for (e.g. ophthalmic artery arising from the middle meningeal artery or accessory middle meningeal artery), as well as potentially dangerous anastomotic vessels (e.g. external carotid to vertebrobasilar system anastamosis).

Since most skull base tumours are supplied by branches of the external carotid system, these vessels should be individu-

From the Department of Radiology. Toronto Western Hospital, University of Toronto, Toronto, Ontario (Drs. Ter Brugge and Chiu) and Radiology Department, Kremlin Bicetre Hospital, University of Paris IX. France (Dr. Lasjaunias)

Reprint requests to: Dr. K.G. Ter Brugge, Department of Radiology, Toronto Western Hospital, 399 Bathurst Street, Toronto, Ontario. Canada M5T 2S8 

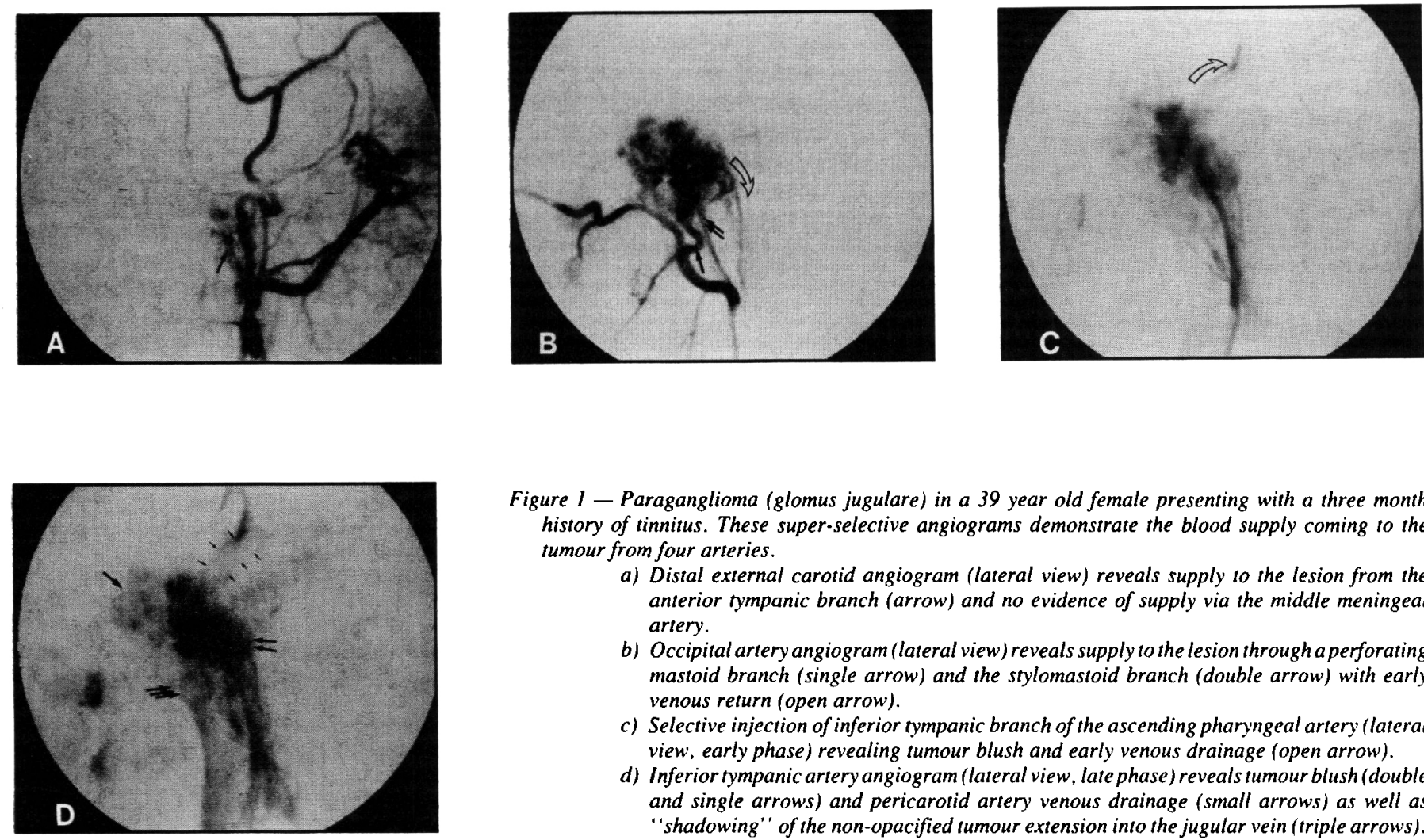

Figure I - Paraganglioma (glomus jugulare) in a 39 year old female presenting with a three month history of tinnitus. These super-selective angiograms demonstrate the blood supply coming to the tumour from four arteries.

a) Distal external carotid angiogram (lateral view) reveals supply to the lesion from the anterior tympanic branch (arrow) and no evidence of supply via the middle meningeal artery.

b) Occipital artery angiogram (lateral view) reveals supplyto the lesion through a perforating mastoid branch (single arrow) and the stylomastoid branch (double arrow) with early venous return (open arrow).

c) Selective injection of inferior tympanic branch of the ascending pharyngeal artery (lateral view, early phase) revealing tumour blush and early venous drainage (open arrow).

d) Inferior tympanic artery angiogram (lateral view, late phase) reveals tumour blush (double and single arrows) and pericarotid artery venous drainage (small arrows) as well as "shadowing" of the non-opacified tumour extension into the jugular vein (triple arrows).

ally catheterized and injected (Figure 1). Frequently, a lesion which apparently receives its main blood supply from the internal carotid system may, in fact, be reached safely for the purpose of embolization through the branches of the external carotid system (Figure 2).

Over the past seven years, more than 600 patients have been embolized by one of the authors (P.L.), including therapeutic angiography in 182 base of skull tumours. This series included 62 patients with juvenile angiofibroma and 49 patients with paragangliomas of the head and neck, which were previously reported. ${ }^{9.10}$ Embolization in combination with surgery has resulted in a significant reduction in blood loss at the time of surgery for the removal of paragangliomas and juvenile angiofibromas, and a low rate of recurrence on long term follow-up..$^{9.10}$ No permanent neurological deficit has occurred following the endovascular treatment, although transient cranial nerve palsies were noted following embolization of individual branches of the external carotid system in a small number of patients. The risk of cranial nerve palsies following embolization is higher if liquid embolic substances are used.

Many skull base tumours, although benign, are locally invasive and tend to recur if they are not removed completely (e.g. juvenile angiofibroma, paraganglioma and basal meningioma). Because of technical difficulties related to the proximity of major blood vessels and cranial nerves, these benign lesions are often treated by radiation therapy rather than by radical surgical resection. In some European centres, however, presurgical

- embolization has been performed routinely and has permitted complete or radical subtotal surgical removal, with a very low rate of recurrence. Some lesions, previously considered in- operable, became operable following embolization, while others which previously would have been considered for radiation therapy were embolized successfully in a combined therapeutic approach (e.g. cavernous sinus meningioma). We would stress the critical importance of a detailed knowledge of the normal vascular anatomy of the region, as well as demonstration of the detailed individual blood supply in each case. Tumour feeders can be catheterized to permit the use of aggressive cytotoxic drugs such as absolute alcohol. Tumour feeders can be blocked, in order to reroute the blood supply to one or two main feeders, thereby increasing the accessibility of the entire tumour to in situ infusion of chemotherapy. In malignant tumours, however, the objective of endovascular treatment is limited to palliation; embolization may diminish pain by decreasing the mass effect of the tumour, or can be used to control hemorrhage which sometimes results from arterial ulceration or postradiation necrosis.

\section{Conclusion}

Basal skull tumours require a multidisciplinary approach in order to provide the best possible treatment for each patient. Super-selective catheterization techniques have made it possible to demonstrate detailed diagnostic information, upon which a treatment protocol can be built. Endovascular embolization techniques, although dependant on the skill and experience of the angiographer, should be considered as a presurgical treatment modality for benign lesions of the skull base, and as alternate therapy for non-operable benign or malignant lesions. 

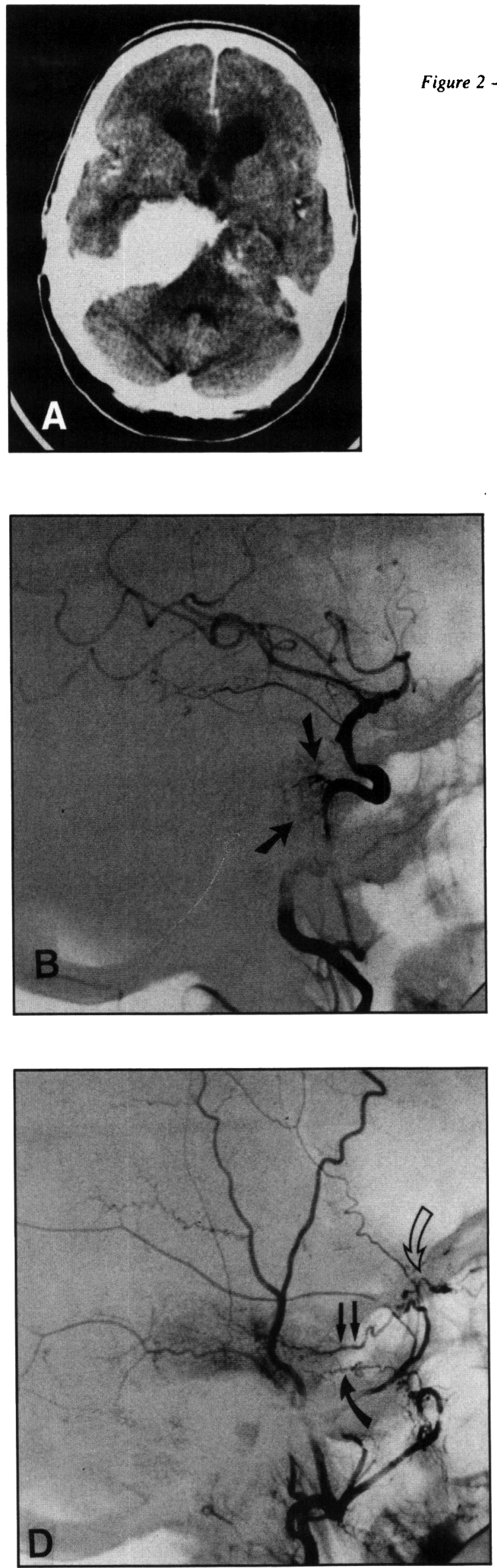

Figure 2 - Meningioma: A 47-year-old male presented a four month history of headaches and seizures. a) Enhanced CT scan showing large well defined tumour arising from petrous apex and extending upwards, causing displacement of the midbrain, and ventricular enlargement.

b) Right internal carotid artery angiogram (lateral view, early phase) shows supply to the tumour from the C4 portion of the internal carotid artery [ICA] (arrows), as well as localized mass effect.

c) Right internal carotid artery angiogram (lateral view, late phase) reveals tumour blush and therefore the extent of lesion supplied by ICA (arrows).

d) Distal external carotid artery angiogram (lateral view, early phase) reveals supply to the lesion via the artery of the foramen rotundum (single arrow) and the artery of the free margin of the tentorium (double arrows), which in this case arises from the middle meningeal artery. Also note the potentially dangerous anastomosis with the orbit (open arrows).

e) Distal external carotid angiogram (lateral view, late phase) reveals extent of tumour blush to be similar to that at the time of the internal carotid artery injection (arrows).
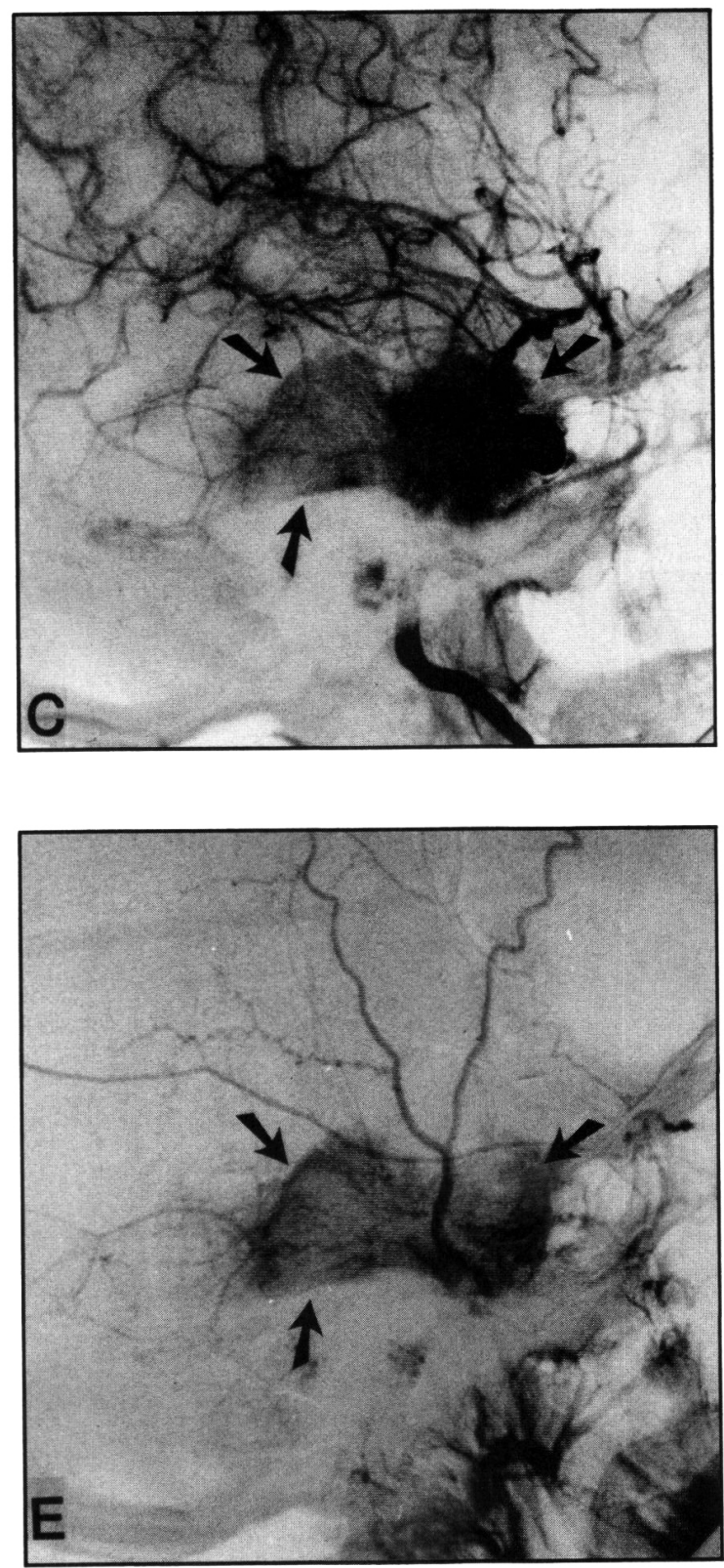


\section{REFERENCES}

I. Bergeron RT, Osborn AG, Som PM. Head and neck imaging, excluding the brain. St. Louis: Mosby Company, 1984.

2. Bohman L, Mancuso A, Thompson J et al. CT approach to benign nasopharyngeal masses. Am J Radiol 1981; 136:173-180.

3. Bryan RN. Sessions RB, Horowitz BL. Radiographic management of juvenile angiofibromas. Am J Neurorad 1981; 2:157-166.

4. Leekham R, Ter Brugge KG, Chiu MC. Computed tomography of the pterygopalatine fossa. J Canad Assoc Radiol 1981: 32:97-100.

5. Osborn AG. Radiology of the pterygoid plates and pterygopalatine fossa. Am J Radiol 1979; 132:389-394.

6. Valvanis A. Fisch U. The contribution of computed tomography to the management of glomus tumours of the temporal bone. Revue de Laryngol 1983: 104:411-415.
7. Lasjaunias P. Cranio-facial and upper cervical arteries: Collateral circulation and angiographic protocol. Baltimore: Williams and Wilkins, 1983.

8. Lasjaunias P. Cranio-facial and upper cervical arteries: Anatomy, normal angiographic aspects. Baltimore: Williams and Wilkins, 1981.

9. Lasjaunias P, Picard L, Manelfe $\mathrm{C}$ et al. Juvenile angiofibroma, review of $S 3$ cases embolized. J Neurorad 1980; 7:73-95.

10. Lasjaunias P. Branchial paragangliomas: review of 40 cases embolized between 1977 and 1984. J Fran d'orc (in press). 